TITLE:

\title{
Fluctuation effects in underdoped cuprate superconductors under a magnetic field
}

\author{
$\operatorname{AUTHOR}(\mathrm{S}):$ \\ Ikeda, R
}

CITATION:

Ikeda, R. Fluctuation effects in underdoped cuprate superconductors under a magnetic field. PHYSICAL REVIEW B 2002, 66(10): 100511.

ISSUE DATE:

2002-09-01

URL:

http://hdl.handle.net/2433/49973

RIGHT:

Copyright 2002 American Physical Society 
PHYSICAL REVIEW B 66, 100511(R) (2002)

\title{
Fluctuation effects in underdoped cuprate superconductors under a magnetic field
}

\author{
Ryusuke Ikeda \\ Department of Physics, Kyoto University, Kyoto 606-8502, Japan
}

(Received 26 June 2002; published 30 September 2002)

\begin{abstract}
Fluctuation effects in underdoped cuprates under high fields are examined by trying to fit theoretical results to resistivity and Nernst data in vortex states. The superconducting (SC) fluctuation in underdoped cuprates includes not only the ordinary thermal contribution but also a large amount of quantum dynamical contributions. Together with this, the presence of a SC pseudogap region $T_{0}-T_{c 0}$ increasing with underdoping is found to be the origin of the Nernst coefficient becoming anomalously smaller and the in-plane coherence length apparently increasing with underdoping.
\end{abstract}

DOI: 10.1103/PhysRevB.66.100511

PACS number(s): 74.40.+k, 74.20.De, 74.72.-h, 74.60.-w

It has been well understood that the field-induced fanshaped broadenings of curves of resistivity and thermodynamic quantities, typically seen in optimally (hole-)doped high- $T_{c 0}$ cuprate superconductors (HTS's), are thermal superconducting (SC) fluctuation phenomena mainly in the vortex liquid region of the normal phase below the zero-field $(H=0)$ transition point $T_{c 0} \cdot{ }^{1}$ In applied magnetic fields of a tesla range perpendicular to the SC planes, the in-plane resistivity and other physical quantities in these materials show familiar behaviors ${ }^{2,3}$ correlated with one another. For instance, the onset temperature of the fluctuation effects suggested from resistive data is almost the same as the corresponding one of thermodynamic and thermomagnetic data. This familiar correlation is typically seen in much lower fields than $H_{c 2}(0)$, where the fluctuation is purely thermal, ${ }^{2}$ and the quantum fluctuation contribution is negligible.

In contrast, the resistivity in other HTS's with lower $T_{c 0}$ often behaves in an uncorrelated manner with thermodynamic quantities. Typically, as the applied field is higher, resistivity data in electron-doped HTS's and some of over (hole-)doped materials show not a fan shaped ${ }^{1}$ but a flat curve $^{4,5}$ following the in-plane normal resistivity $\rho_{n}(T)$ $=\left[\sigma_{n}(T)\right]^{-1}$ curve until a vortex-glass $(\mathrm{VG})$ transition field, lying much below ${ }^{5,6}$ an effective $H_{c 2}(T)$ determined thermodynamically, is approached from above. In over (hole)-doped materials ${ }^{4}$ with high $\sigma_{n}$-value $\left[\simeq 10^{2}\left(R_{q} s\right)^{-1}\right]$, such an absence of correlation is not surprising because the fluctuation conductivity $\sigma_{f}$ is negligible compared with $\sigma_{n}$ in the total conductivity $\sigma=\sigma_{n}+\sigma_{f}$ over a wide temperature range, where $R_{q}=6.45(\mathrm{k} \Omega)$ is the resistance quantum measured, and $s \simeq 10($ A) is a typical size of the spacing between SC layers. However, the corresponding uncorrelated behavior seen in the electron-doped materials ${ }^{5}$ with $\sigma_{n}$ of the same order as in the optimally doped $\mathrm{YBCO},{ }^{3}$ is intrinsic, and its origin needs to be attributed to a fluctuation property. Similar behaviors have also been found in overdoped $\mathrm{La}_{2-x} \mathrm{Sr}_{x} \mathrm{CuO}_{4}$ $(\mathrm{LSCO} x)$ (Ref. 7) and $\kappa$-(ET) ${ }_{2}$ organic superconductors. ${ }^{8,9}$ As argued elsewhere ${ }^{10}$ by fitting to data, ${ }^{9}$ the main origin is expected to consist in the quantum dynamical nature, enhanced with increasing the field, of the SC fluctuation. In general, $\sigma_{f}$ defined in a Ginzburg-Landau (GL) theory decreases $^{11}$ as the SC fluctuation is dominated not by the thermal fluctuation but rather by a quantum dynamical fluctuation. Such an absence of correlation in the quantum re- gime near $T=0$ between the resistivity and the magnetization was predicted in Ref. 11.

Recent data of resistivity and Nernst coefficient in underdoped cuprates ${ }^{7,12}$ have also shown similar high field behaviors, suggesting a large quantum fluctuation effect. The twodimensional (2D) field-tuned superconductor-insulator transition (FSIT) behavior, seen in resistance data in strongly underdoped cases, ${ }^{12-14}$ cannot occur without the quantum nature of SC fluctuation, ${ }^{11,15}$ and, as the SC fluctuation is enhanced, the quantum contribution to the fluctuation dominates over the thermal one. Hence it is natural to expect the $\mathrm{SC}$ fluctuation effect to be stronger with underdoping. However, a sharp drop of resistivity in high fields, which often appears even in underdoped materials, was regarded as a mean-field-like behavior in the literature. ${ }^{16}$ Further, the resistance data in the pseudogap regime suggest an in-plane coherence length increasing ${ }^{17}$ with underdoping.

In this paper, we try to improve the understanding of SC fluctuation properties in underdoped cuprates within the GL theory, and argue that, together with a quantum fluctuation, a large width $T_{0}-T_{c 0}$ of a SC pseudogap region is a key factor for consistently explaining the conflicting observations in underdoped cuprates, where $T_{0}$ is the mean-field transition temperature in $H=0$. We start with the 2D GL action expressed in terms of a single-component pair field $\psi(\mathbf{r}, \tau)$,

$$
\begin{aligned}
S= & \int_{\mathbf{r}}\left[\beta \sum_{\omega}\left[\psi_{\omega}(\mathbf{r})\right]^{*} \gamma\left(\mathbf{Q}^{2}\right)|\omega| \psi_{\omega}(\mathbf{r})\right. \\
& \left.+\int_{0}^{\beta} d \tau\left([\psi(\mathbf{r}, \tau)]^{*} \mu\left(\mathbf{Q}^{2}\right) \psi(\mathbf{r}, \tau)+\frac{b}{2}|\psi(\mathbf{r}, \tau)|^{4}\right)\right]
\end{aligned}
$$

$\left(\hbar, k_{B}=1\right)$, where $\psi(\tau)=\Sigma_{\omega} \psi_{\omega} e^{-i \omega \tau}, \beta$ the inverse temperature, $\tau$ the imaginary time, and $b>0$. The 3D nature due to the coupling between SC planes will be included later in considering a VG contribution. When the GL approach is applied to the low- $T$, high- $H$ region, $H$ dependences of the coefficients $\gamma, \mu$, and $b$ need to be taken into account since the familiar low- $T$ divergences of these coefficients in $H$ $=0$ clean limit are cut off by the orbital depairing effect of the magnetic field (for simplicity, the Pauli paramagnetic depairing effect, becoming important in stronger fields, will be 
neglected together with a particle-hole assymmetric dynamical term leading to a fluctuation Hall effect). In fact, the coefficients $\gamma$ and $\mu$ are functionals of the gauge-invariant gradient $\mathbf{Q}=-\mathrm{i} \nabla+2 \pi \mathbf{A} / \phi_{0}$ and, once $\psi$ is decomposed into the Landau levels (LL's), are replaced by the coefficients $\gamma_{n}$ and $\mu_{n}$ dependent on the LL index $n$. Hereafter, the familiar clean limit ${ }^{18}$ will be invoked to describe these coefficients in a form reasonable even in low $T$ and high $H$. Then $\gamma_{n}$ and $\mu_{n}$ are given by

$$
\begin{gathered}
\gamma_{n}=\frac{\beta}{2 \pi} \int_{0}^{\infty} d s \frac{s}{\sinh (s)} L_{n}\left(u_{c}^{2} s^{2}\right) e^{-\left(u_{c} s\right)^{2} / 2}, \\
\mu_{n}=\ln \left(\frac{T}{T_{0}}\right)+\int_{0}^{\infty} d s \frac{1-L_{n}\left(u_{c}^{2} s^{2}\right) e^{-\left(u_{c} s\right)^{2} / 2}}{\sinh (s)},
\end{gathered}
$$

respectively, where $u_{c}=T_{0} \sqrt{H /\left(2 H_{0} e^{\gamma}\right)} / T, H_{0}$ is the $T=0$ value of the mean field upper critical field $H_{0}(T)$ measuring the in-plane coherence length, $L_{n}(x)$ is the $n$th order Laguerre polynomial, and $\gamma=0.5771$ is the Euler constant. Although, in $d_{x^{2}-y^{2}}$ pairing, cross terms between the lowest LL and the $n=4 m(m \geqslant 1)$ higher LL's arise in the quadratic terms of Eq. (1), they can be safely neglected in situations of our interest where the lowest LL mode is dominant. The time scales $\gamma_{2 m+1}$ vanish in the low- $T$ limit, and are highly sensitive to $T$ and $H$ in contrast to $\gamma_{0}$, which takes values close to 0.3 in the field and temperature ranges we have examined. Other material and doping dependences will be assumed to be included in the coefficient $b$ from which, in low- $H$ limit, the $T=0$ magnetic penetration depth $\lambda(0)$ is defined. For instance, (if any) effects of other competing order parameter fluctuations ${ }^{19}$ may be seen as having been integrated out and absorbed into $b$. Further, a numerical computation of $b$ consistent with Eqs. (2) suggests that its $H$ and $T$ dependences are similar to those of $\gamma_{0}$. For these reasons, $b$ will be treated as one of fitting parameters independent of $H$ and $T$.

To renormalize the $\psi$ fluctuation, the lowest LL approximation will be used. Following previous works, ${ }^{1,11}$ the renormalized mass parameter $\mathcal{G}_{0}(0)$ defined through the propagator $\mathcal{G}_{0}(\omega)=\left\langle\left|\varphi_{0}(\omega)\right|^{2}\right\rangle=\left(\gamma_{0}|\omega|+\left[\mathcal{G}_{0}(0)\right]^{-1}\right)^{-1}$ for the lowest LL fluctuation field $\varphi_{0}$ is written as

$$
\mathcal{G}_{0}(0)=1 /\left(\mu_{0}+\Delta \Sigma_{\mathrm{h}}+\Sigma_{0}+\Delta \Sigma_{1}\right) \text {. }
$$

The main roles of $\mu_{0}$ renormalization are played by the Hartree term $\Sigma_{0}$, which is expressed as

$$
\begin{aligned}
\Sigma_{0}= & \frac{16 \pi^{2} \lambda^{2}(0)}{\phi_{0}^{2} s \beta} \frac{H}{H_{0}} \sum_{\omega} \mathcal{G}_{0}(\omega) \\
= & \frac{16 \pi \lambda^{2}(0)}{\phi_{0}^{2} s \gamma_{0}} \frac{H}{H_{0}} \int_{0}^{\epsilon_{c}} d \epsilon \\
& \times \operatorname{coth}\left(\frac{\beta \epsilon}{2 \gamma_{0}}\right) \frac{\epsilon}{\epsilon^{2}+\left[\mathcal{G}_{0}(0)\right]^{-2}},
\end{aligned}
$$

where the cutoff $\epsilon_{c}$ is a constant of order unity, and the coefficient $b$ was replaced with the familiar GL expression ${ }^{1}$
$16 \pi^{2} \lambda(0)^{2} /\left(\phi_{0} H_{0}\right)$. Although other renormalization (correction) term $\Delta \Sigma_{l}$ within the lowest LL [see, for instance, Eq. (2.11) of Ref. 11] should be included, it is not essential to our semiquantitative comparison with the data and may be dropped hereafter. The term $\Delta \Sigma_{h}$ expressing a sum of higher LL contributions is insensitive to $H$ at least in $H \ll H_{0}$ and can be regarded as contributing to a shift of $H=0$ transition temperature in $\mu_{0}$. Then $\Delta \Sigma_{h}$ may be written ${ }^{20}$ as $\ln \left(T_{0} / T_{c 0}\right)$, and the effective upper critical field $H_{c 2}^{*}(T)$, defined consistently with $T_{c 0}$, is determined in the clean limit by $\mu_{0}$ $+\Delta \Sigma_{h}=0$ and takes the form

$$
H_{c 2}^{*}(T)=H_{0}\left(\frac{T_{c 0}}{T_{0}}\right)^{2} \Phi(t),
$$

while $H_{0}(T)=H_{0} \Phi\left(T / T_{0}\right)$, where $t=T / T_{c 0}$, and the function $\Phi(x)$ satisfies $\Phi(0)=1$ and $\Phi(1)=0$. If $1-T_{c 0} / T_{0}$ $\ll 1$, as in optimally doped $\mathrm{YBCO},{ }^{1}$ the presence of the parameter $\left(T_{c 0} / T_{0}\right)^{2}$ is unimportant in Eq. (5), and physical properties below $T_{c 0}$ may be described without distinguishing $T_{c 0}$ from $T_{0}$. However, in cases with a large $T_{0} / T_{c 0}$, this parameter significantly affects fluctuation phenomena in nonzero fields.

Now, let us consider transport quantities. Although, in addition to the lowest LL mode, the $n=1$ LL mode and the in-plane electric current (EC) vertices need to be examined to obtain $\sigma_{f}$ and the transport entropy $s_{\phi}$, microscopic consideration on $\sigma_{f}$ can be avoided as follows. As shown previously, ${ }^{15}$ the mean-field vortex flow property requires that, irrespective of microscopic details, the $n=1$ renormalized mass parameter $\mathcal{G}_{1}(0)$ below $H_{c 2}^{*}(T)$ should be given by a factor accompanying the EC vertex. On the other hand, since $\mathcal{G}_{1}(0)$ below $H_{c 2}^{*}(T)$ is well approximated by $\left(\mu_{1}\right.$ $\left.-\mu_{0}\right)^{-1}$ (Ref. 20) insensitive to $T$ at low $T$, the EC vertex is found without microscopic calculations. Then $\sigma_{f}$ calculated in terms of the Kubo formula consistently with Eq. (3) is ${ }^{11}$

$$
\begin{aligned}
s R_{q} \sigma_{f}= & \frac{\gamma_{0}}{2\left[\mathcal{G}_{1}(0)\right]^{2} \beta} \sum_{\omega}\left[\mathcal{G}_{0}(\omega) \mathcal{G}_{1}(\omega)\left[\mathcal{G}_{0}(\omega)+g \mathcal{G}_{1}(\omega)\right]\right. \\
& \left.-\frac{\left[\mathcal{G}_{0}(\omega)\right]^{2}+g^{2}\left[\mathcal{G}_{1}(\omega)\right]^{2}}{\left[\mathcal{G}_{1}(0)\right]^{-1}+g\left[\mathcal{G}_{0}(0)\right]^{-1}}\right]
\end{aligned}
$$

where $g=\gamma_{1} / \gamma_{0}$, and $\mathcal{G}_{1}(\omega)=\left(\gamma_{1}|\omega|+\left(\mathcal{G}_{1}(0)\right)^{-1}\right)^{-1}$. It is easily seen that, in the quantum $(T \rightarrow 0)$ limit, Eq. (6) vanishes $^{11}$ and that, in the opposite thermal limit with no $\omega$ $\neq 0$ terms, Eq. (6) is independent of $\mathcal{G}_{1}(0)$ due to the relation $g \mathcal{G}_{1}(0) \ll \mathcal{G}_{0}(0)$. Further, we numerically verified that, even if the $\omega \neq 0$ terms are included, this cancellation on $\mathcal{G}_{1}(0)$ works extremely well particularly in higher $H$.

On the other hand, $s_{\phi}$ is, by definition, ${ }^{21}$ proportional to the heat current vertex, which may have a strong $T / T_{0}$ dependence of electronic origins. For brevity, hereafter we use the GL expression ${ }^{21}$ of the heat current and will not consider the very low $T / T_{0}$ region in which $s_{\phi}$ decreases ${ }^{22}$ upon cooling independently of $\sigma$ (see the figures). Consistently with Eq. (6), $s_{\phi}$ is obtained in terms of a Kubo formula and, using $g \mathcal{G}_{1}(0) \ll \mathcal{G}_{0}(0)$, is simplified as 

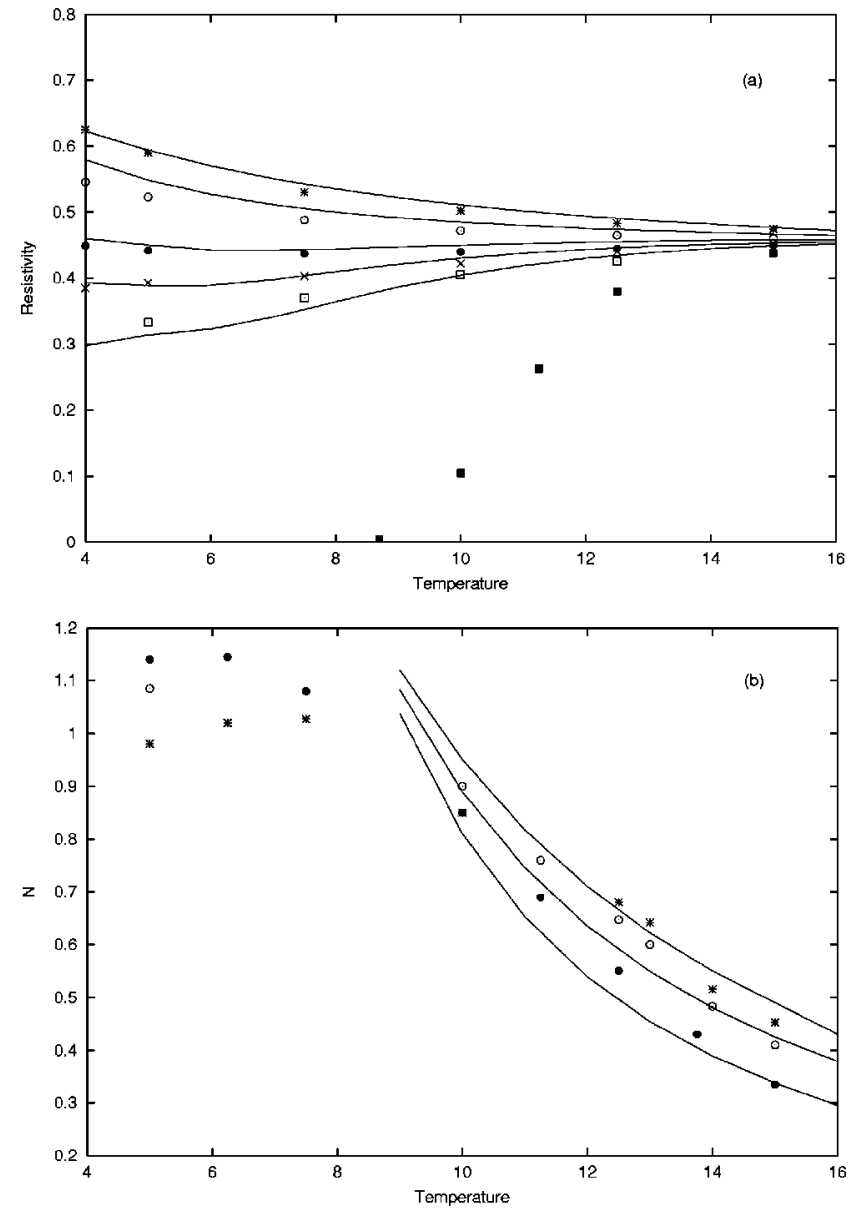

FIG. 1. (a) Resistivity $\rho(\mathrm{m} \Omega \mathrm{cm})$ and (b) Nernst coefficient $N(\mu \mathrm{V} / \mathrm{K})$ data in LSCO $x=0.06$ (Ref. 23) in 2 (open square), 3 (cross), 4 (closed circle), 6 (open circle), and 8 (asterisk) (T) at each $T(\mathrm{~K})$ and the corresponding theoretical (solid) curves. The parameter values used are $\lambda(0)=2.3(\mu \mathrm{m}), \quad H_{0}=493(\mathrm{~T}), \quad T_{c 0}$ $=13(\mathrm{~K}), s=1.5(\mathrm{~nm})$, and $T_{0}=96(\mathrm{~K})$.

$$
s_{\phi} \simeq \frac{H}{H_{0} s \mathcal{G}_{1}(0)} \sum_{\omega} \mathcal{G}_{1}(\omega) \mathcal{G}_{0}(\omega) \simeq \frac{\phi_{0}^{2}}{16 \pi^{2} \lambda^{2}(0) T} \Sigma_{0}
$$

where the factor $\left(\mathcal{G}_{1}(0)\right)^{-1}$ is carried by an ET vertex. That is, in the lowest LL approximation, $s_{\phi}$ in the GL region is proportional to the fluctuation entropy even in the quantum case, and the mean-field result $\phi_{0}^{2} \beta(1$ $\left.-T / T_{c 0}\right) /\left[16 \pi^{2} \lambda^{2}(0)\right]$ is recovered when both $H$ and $T$ are lowered enough.

We have tried to fit theoretical curves following from Eqs. (6) and (7) to the resistivity $\rho$ and Nernst signal data in LSCO $x$ samples with $x=0.06$ (Ref. 23) and $0.08,{ }^{12}$ and the results are given in Figs. 1 and 2, respectively, where the Nernst coefficient $N=\rho s_{\phi} / \phi_{0}$. The used values of material parameters are shown in the figure captions. Since, by definition, our $T_{c 0}$ in two dimensions corresponds to a (amplitude-) fluctuation-corrected BCS critical temperature denoted in Ref. 24 as $T_{c}^{0}, T_{c 0}$ was identified in the figures with the onset of a remarkable resistivity drop in $H=0$. The normal conductivity $\sigma_{n}$ is assumed to take the empirical form const $/ \ln \left(T_{p} / T\right)$ (Ref. 25) with $T_{p} \gg T_{c 0}$. Regarding the
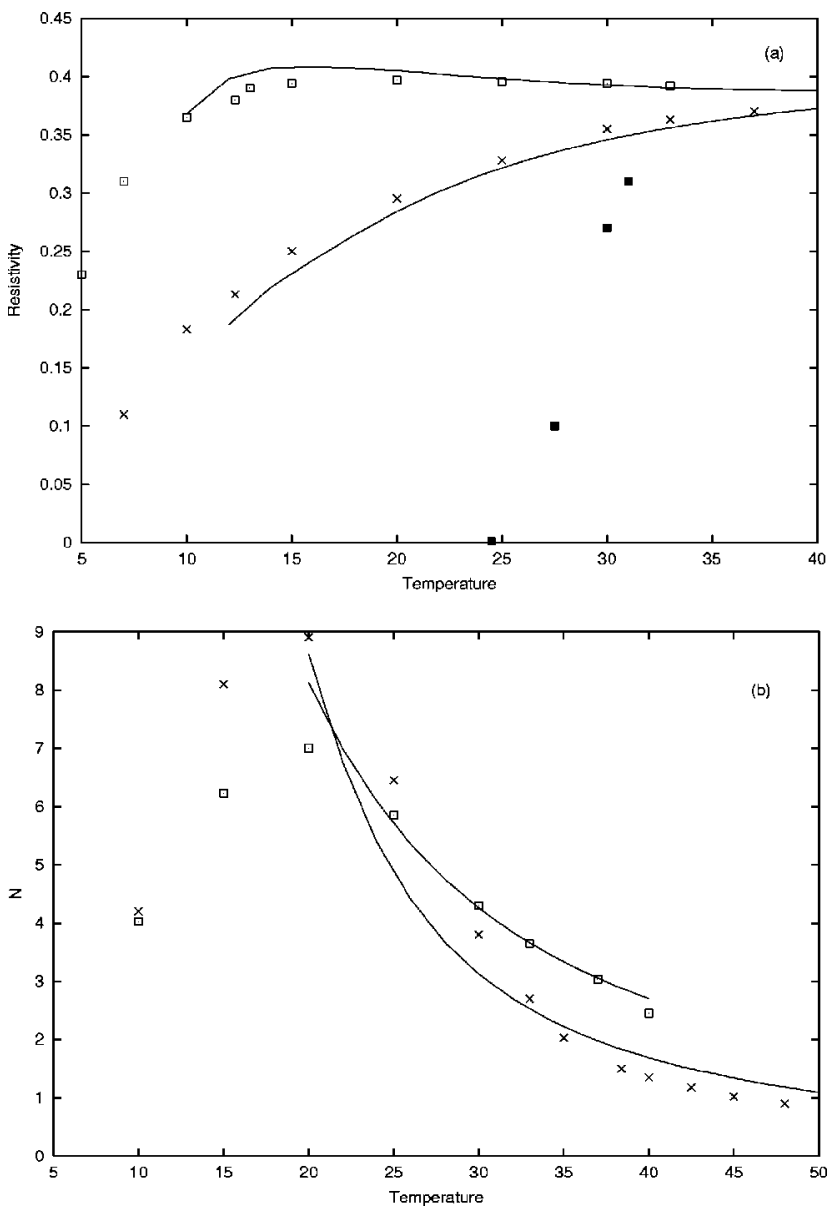

FIG. 2. Corresponding results to Fig. 1 for LSCO $x=0.08$ (Ref. 12) in 12 and $26(\mathrm{~T})$. The parameter values are $\lambda(0)$ $=0.43(\mu \mathrm{m}), H_{0}=235(\mathrm{~T}), T_{c 0}=32(\mathrm{~K}), s=1.5(\mathrm{~nm})$, and $T_{0}$ $=96(\mathrm{~K})$.

VG fluctuation term $\sigma_{v g}$, added to $\sigma$ for describing the low$T$ tails of $\rho$ curves, a 3D form $\sigma_{v g}=0.01\left(R_{q} s\right)^{-1} \gamma_{0} T_{c 0} /(t$ $\left.-t_{g}\right)^{4}$ was assumed in Fig. 2 with $t_{g}=0.125(0.016)$ for 12 (26) $(\mathrm{T})$, while a $2 \mathrm{D}$ form $^{15}$ used in analyzing the FSIT behaviors in the $s$-wave pairing case was applied in Fig. 1 by assuming a vortex pinning strength as a fitting parameter independent of $\lambda(0)$. Details of such an analysis of FSIT behaviors will be explained elsewhere. ${ }^{10}$ Here we simply note that the detailed forms of $\sigma_{v g}$ are inessential to our main conclusion given below. For instance, the flat $\rho$ curves near 4 (T) in Fig. 1 are created mainly by a quantum behavior ${ }^{11}$ shown by Eq. (6), and the $\sigma_{v g}$ contribution was quantitatively negligible there.

Although there is a slight disagreement in the $T$ dependences of $N$ between the data and theoretical curves, we feel that the fitting to the data is satisfactory when taking account of the use of our simplified model with a reduced number of fitting parameters. The figures show an enhancement of quantum SC fluctuation accompanying the underdoping. In Fig. $2, H_{c 2}^{*}(0)$ is close to $26(\mathrm{~T})$, while $H_{c 2}^{*}[4(\mathrm{~K})] \simeq 8(\mathrm{~T})$ and $H_{c 2}^{*}[7(\mathrm{~K})] \simeq 6(\mathrm{~T})$ in Fig. 1 so that the resistance may show an insulating behavior even below $H_{c 2}^{*}(\mathrm{~T})$. Further, the fitting results imply the following doping dependences of 
material parameters. First, $\lambda(0)$ significantly increases with underdoping and, in the $x=0.06$ case, is longer than $1(\mu \mathrm{m})$. Judging from the LSCO data, ${ }^{26}$ the $\lambda(0)$ values used in the figures are not unreasonable. Second, through the doping dependence of $H_{0}$, the in-plane coherence length $d e$ creases with underdoping in contrast to the experimental estimation. ${ }^{17}$ Further, the SC pseudogap region measured by $T_{0}-T_{c 0}$ was assumed to become wider with underdoping. It is unclear whether the obtained $T_{0}$ value should be quantitatively compared with, for instance, the onset temperature $T_{\nu}$ (Ref. 27) of the Nernst effect. Actually, the $T_{0}=96(\mathrm{~K})$ in Fig. 2 was estimated from the data for larger $H / H_{0}$ values than in Fig. 1, and its actual value may be slightly higher. Thus a doping dependence of $T_{0}$ suggested through the figures does not necessarily contradict $T_{v}$ in LSCO,${ }^{27}$ decreasing with underdoping in $x \leqslant 0.1$.

It is important to notice that the $\sigma_{f}$ expression of Eq. (6) is invariant under the replacement of parameters $\lambda(0)$ $\rightarrow \lambda(0) T_{c 0} / T_{0}, H_{0} \rightarrow H_{c 2}^{*}(0)$, and $T_{0} / T_{c 0} \rightarrow 1$. That is, the presence of a large SC pseudogap is not uncovered by examining only the magnetoresistance data, and a neglect of SC pseudogap region would lead to a much shorter penetration depth and an in-plane coherence length growing ${ }^{17}$ with underdoping. More importantly, as a result of the much shorter penetration depths, the assumption $T_{0}=T_{c 0}$ leads to $N$ values in the $x=0.08$ case which are one order of magnitude larger than the data in Fig. 2, and to $N$ values in the $x=0.06$ case which are two orders of magnitude larger than in Fig. 1. It is quite difficult to resolve such a serious discrepancy, for instance, simply by improving the prefactor of the heat current.
This result, requiring $T_{0}-T_{c 0}$ to increase with underdoping, agrees with the opinion that $T_{\nu}$, much higher than $T_{c 0}$, is essentially identical to the mean field transition point $T_{0}$ or $H_{0}(T)$.

Recently, Wang et al. ${ }^{7}$ argued that a field $H^{*}$ at which the Nernst coefficient reaches its maximum is, in their overdoped LSCO, due to a simulatneous sharp drop of $\rho$ related to an effectively longer vortex core size. From Fig. 1, such a mean-field argument on the sharp drop of $\rho$ is generally invalid, since a picture based on an anomalous feature near the vortex cores would become more applicable with underdoping, while a comparison between the $\rho$ and $N$ data in high fields in Fig. 1 clearly shows that the decrease of $N$ at lower temperatures is due not to $\rho$ but to $s_{\phi}$. As mentioned in the introduction, a sharp drop of $\rho$ much below $H_{c 2}^{*}(T)$ $\left[\leqslant H_{0}(T)\right]$ occurs even in organic materials ${ }^{9,10}$ and is due not to a mechanism peculiar to the cuprates but to a $3 \mathrm{D}$ VG transition in systems with a large quantum SC fluctuation.

In conclusion, the resistance and Nernst data in underdoped LSCO were consistently explained to clarify the doping dependences of fluctuation effect and of SC parameters. The in-plane coherence length was argued to decrease with underdoping. To explain those transport data consistently, microscopic details near the vortex cores are not necessary, and taking account of the quantum SC fluctuation and a SC pseudogap region $T_{0}-T_{c 0}$, both of which are enhanced with underdoping, is indispensable.

The author thanks C. Capan and W. Lang for sending him their unpublished data and for useful discussions.
${ }^{1}$ R. Ikeda, T. Ohmi, and T. Tsuneto, J. Phys. Soc. Jpn. 60, 1051 (1991); Phys. Rev. Lett. 67, 3874 (1991).

${ }^{2}$ U. Welp et al., Phys. Rev. Lett. 67, 3180 (1991).

${ }^{3}$ H.-C. Ri et al., Phys. Rev. B 50, 3312 (1994).

${ }^{4}$ A.P. Mackenzie et al., Phys. Rev. Lett. 71, 1238 (1993).

${ }^{5}$ S. Kleefisch et al., Phys. Rev. B 63, 100507 (2001), and references therein.

${ }^{6}$ A. Carrington, A.P. Mackenzie, and A. Tyler, Phys. Rev. B 54, R3788 (1996).

${ }^{7}$ Y. Wang et al., Phys. Rev. Lett. 88, 257003 (2002).

${ }^{8}$ H. Ito et al., Physica B 201, 470 (1994).

${ }^{9}$ T. Sasaki (private communication); M. Lang et al., Phys. Rev. B 49, 15227 (1994).

${ }^{10} \mathrm{R}$. Ikeda, written for Proc. of LT23 and (unpublished).

${ }^{11}$ R. Ikeda, Int. J. Mod. Phys. B 10, 601 (1996).

${ }^{12}$ C. Capan et al., Phys. Rev. Lett. 88, 056601 (2002).

${ }^{13}$ G.T. Seidler, T.F. Rosenbaum, and B.W. Veal, Phys. Rev. B 45, 10162 (1992).

${ }^{14}$ K. Karpinska et al., Phys. Rev. Lett. 77, 3033 (1996).

${ }^{15}$ H. Ishida and R. Ikeda, J. Phys. Soc. Jpn. 71, 254 (2002).
${ }^{16}$ See, for instance, D.J.C. Walker et al., Phys. Rev. B 51, 9375 (1995).

${ }^{17}$ Y. Ando and K. Segawa, Phys. Rev. Lett. 88, 167005 (2002).

${ }^{18} \mathrm{See}$, for instance, the Appendix in P.A. Lee and M.G. Payne, Phys. Rev. B 5, 923 (1972).

${ }^{19}$ S.A. Kivelson et al., cond-mat/0205228 (unpublished).

${ }^{20}$ R. Ikeda, J. Phys. Soc. Jpn. 64, 1683 (1995).

${ }^{21}$ R.J. Troy and A.T. Dorsey, Phys. Rev. B 47, 2715 (1993).

${ }^{22} \mathrm{On}$ this issue in the $s$-wave pairing case, see K. Maki, Physica (Utrecht) 55, 125 (1971).

${ }^{23}$ C. Capan (private communication). Regarding $x=0.06$ case, we have prefered the refined data shown in Fig. 1 to those in Ref. 12 with a very broad resistive transition in $H=0$.

${ }^{24}$ B.I. Halperin and D.R. Nelson, J. Low Temp. Phys. 36, 599 (1979).

${ }^{25}$ S. Ono et al., Phys. Rev. Lett. 85, 638 (2000).

${ }^{26}$ C. Panagopoulos et al., cond-mat/0007158 (unpublished). See Fig. 3 there.

${ }^{27}$ Y. Wang et al., Phys. Rev. B 64, 224519 (2001). 